\title{
Secukinumab Efficacy on Psoriatic Arthritis GRAPPA- OMERACT Core Domains in Patients with or Without Prior Tumor Necrosis Factor Inhibitor Use: Pooled Analysis of Four Phase 3 Studies
}

\author{
Ana-Maria Orbai (D) - M. Elaine Husni · Dafna D. Gladman • \\ Ying Ying Leung $\cdot$ Stefan Siebert · William Tillett · Marijn Vis · \\ Olivier Chambenoit · Xiangyi Meng · Philip J. Mease
}

Received: March 26, 2021 / Accepted: June 14, 2021 / Published online: July 3, 2021

(C) The Author(s) 2021

\begin{abstract}
Background: Psoriatic arthritis (PsA) is a chronic, heterogeneous, immune-mediated disease manifesting as a spectrum of possible inflammatory signs and symptoms. Clinicians need therapeutic choices that work across all active PsA disease domains, as well as practical information about efficacy of available treatments for individual domains in specific groups
\end{abstract}

Supplementary Information The online version contains supplementary material available at https:// doi.org/10.1007/s40744-021-00337-5.

A.-M. Orbai $(\bowtie)$

Division of Rheumatology, Johns Hopkins

University School of Medicine, MFL Center Tower,

Suite 4100, 5200 Eastern Ave, Baltimore, MD 21224, USA

e-mail: aorbai1@jhmi.edu

M. E. Husni

Cleveland Clinic, Cleveland, $\mathrm{OH}$, USA

D. D. Gladman

Krembil Research Institute, Toronto Western

Hospital and University of Toronto, Toronto, ON,

Canada

Y. Y. Leung

Duke-NUS Medical School, Singapore General

Hospital, Singapore, Singapore

S. Siebert

Institute of Infection, Immunity and Inflammation,

University of Glasgow, Glasgow, UK of patients. The objective of this study was to evaluate the effect of prior tumor necrosis factor inhibitor (TNFi) exposure on the efficacy of secukinumab across PsA core domains.

Methods: Data were pooled from 2049 participants with PsA in four phase 3 studies (FUTURE 2-5). Efficacy at week 16 was evaluated for each GRAPPA-OMERACT PsA core domain using nonresponder imputation for musculoskeletal disease activity and Psoriasis Area and Severity Index scores or as-observed data for other outcomes. For each measure, comparisons with placebo were made separately in the TNFi-naive and TNFi-inadequate responder/intolerant (TNF-IR) cohorts.

W. Tillett

University of Bath, Bath, UK

M. Vis

Erasmus Medical Center, Rotterdam, Netherlands

O. Chambenoit · X. Meng

Novartis Pharmaceuticals Corporation, East

Hanover, NJ, USA

P. J. Mease

Swedish Medical Center/Providence St, Joseph

Health and University of Washington, Seattle, WA, USA 
Results: Treatment with secukinumab improved PsA disease activity across all disease domains regardless of previous TNFi use, although TNFi-naive patients experienced numerically greater benefits in most outcomes. Among patients treated with secukinumab $300 \mathrm{mg}, \quad 41.5 \%$ and $24.4 \%$ of TNFi-naive patients $(P<0.05$ vs placebo $)$ and $18.6 \%$ and $9.0 \%$ of TNF-IR patients (nonsignificant vs placebo) experienced resolution in 66 swollen and 68 tender joint counts, respectively; additionally, $37.2 \%$ of TNFi-naive patients and $24.2 \%$ of TNF-IR patients achieved complete resolution of psoriasis at week 16 (all $P<0.05$ vs placebo). Secukinumab effect sizes were generally larger in TNFi-naive vs TNF-IR patients for musculoskeletal and patient-reported domains.

Conclusions: Secukinumab demonstrated efficacy vs placebo across GRAPPA-OMERACT PsA core domains. Higher responses among TNFinaive vs TNF-IR patients suggest that secukinumab should be considered for first-line use in PsA.

\section{PLAIN LANGUAGE SUMMARY}

Psoriatic arthritis (PsA) is a long-term disease that can affect a patient's joints, skin, lower back, physical function, mental health, productivity, and other areas. Drugs called tumor necrosis factor inhibitors (TNFis) can be used to treat PsA, although not all patients benefit from TNFis and many seek other treatment options. These patients, known as TNFi-inadequate responders (TNF-IR), have PsA that is difficult to treat. Another treatment option is secukinumab, a drug that blocks a molecule called interleukin-17 that is involved in PsA. Doctors need to know how different drugs work for treating PsA signs and symptoms in different groups of patients, including TNF-IR patients and those who have never received TNFis (TNFinaive patients). This study used data from 2049 patients in four different PsA clinical trials (FUTURE 2-5) to see how well secukinumab worked at treating different signs and symptoms of PsA in TNFi-naive and TNF-IR patients. After 16 weeks of treatment, patients who took secukinumab saw greater improvements across all measured PsA signs and symptoms than those who took placebo. This was true for both TNFi-naive and TNF-IR patients. TNFi-naive patients seemed to benefit slightly more than TNF-IR patients-especially in their joint symptoms-although this study was not designed to judge the significance of these differences. These results suggest that secukinumab would be an effective first treatment option for patients with PsA. Since secukinumab improves the skin, joints, and other affected areas, it can be useful in treating the whole patient who has psoriatic disease.

Keywords: Psoriatic arthritis; Spondyloarthritis; GRAPPA-OMERACT domains; Secukinumab; TNF-IR; TNFi-naive

\section{Key Summary Points}

In this analysis of pooled phase 3 studies in psoriatic arthritis, secukinumab improved disease burden as measured by the GRAPPA-OMERACT domains, regardless of previous tumor necrosis factor inhibitor (TNFi) exposure

Higher responses in the TNFi-naive vs TNFi-inadequate responder/intolerant (TNF-IR) cohorts, especially in musculoskeletal manifestations and patient-reported outcomes, suggest value of secukinumab as a first-line biologic therapy for the treatment of psoriatic arthritis

Significant improvements in psoriasis and psoriasis-related patient-reported outcomes in both TNFi-naive and TNFi-IR patients receiving secukinumab $300 \mathrm{mg}$ vs placebo suggest that secukinumab is an effective treatment option throughout the course of psoriatic disease 


\section{DIGITAL FEATURES}

This article is published with digital features, including a summary slide and plain language summary to facilitate understanding of the article. To view digital features for this article go to https://doi.org/10.6084/m9.figshare. 14779812 .

\section{INTRODUCTION}

Psoriatic arthritis (PsA) is a rheumatologic disease that affects up to $30 \%$ of patients with psoriasis and up to $0.25 \%$ of all people in the USA [1]. PsA manifests with peripheral joint inflammation, enthesitis, dactylitis, and axial involvement, either alone or in combination, in addition to skin and nail psoriasis [2]. As a result of the heterogeneity of the disease, clinicians need therapeutic choices that work across all active PsA disease domains, as well as practical information about efficacy of available treatments for each individual domain and in specific groups of patients. Previous analyses of relationships between baseline characteristics and treatment response have found that prior tumor necrosis factor inhibitor (TNFi) exposure is the most important observable baseline characteristic $[3,4]$. Patients with TNFi experience are viewed as having disease that is more difficult to treat [5].

Secukinumab is a fully human monoclonal antibody targeting interleukin (IL)-17A. It is approved for the treatment of psoriasis [6-8], PsA $[9,10]$, ankylosing spondylitis (AS) [11], and nonradiographic axial spondyloarthritis[12] and has been shown to improve disease burden in patients with PsA across all PsA core domains in a mixed population of TNFi-naive patients and patients with an inadequate response to TNFis (TNF-IR) [13].

In this study, we explored the effect of previous TNFi exposure on the efficacy of secukinumab as measured by responses across PsA domains, as defined by international consensus through the Group for Research and Assessment of Psoriasis and Psoriatic Arthritis (GRAPPA) and endorsed by Outcome Measures in Rheumatology (OMERACT) [14, 15], using data pooled from four phase 3 studies of patients with PsA [16-19].

\section{METHODS}

\section{Study Population}

This post hoc analysis included patients with active PsA who participated in the phase 3 randomized controlled trials FUTURE 2 (NCT01649375; $\quad N=397) \quad$ [16], $\quad$ FUTURE 3 (NCT01989468; $\quad N=414) \quad$ [17], $\quad$ FUTURE 4 (NCT02294227; $N=341$ ) [18], and FUTURE 5 (NCT02404350; $N=996$ ) [19]. This pooled population consisted of a mixed population of TNFi-naive and TNF-IR patients; TNF-IR patients must have experienced an inadequate response to previous or current TNFi treatment given at the approved dose for at least 3 months or have stopped treatment with at least one TNFi because of safety or tolerability issues. At the time of this analysis, these studies constituted all available data for secukinumab at the approved doses in patients with PsA. The FUTURE 1 study was excluded from this secondary analysis because the intravenous loading dose is not licensed for use in PsA [9]. Data were pooled from these studies according to the following treatment arms at the end of the 16-week, double-blind, placebo-controlled period: patients received either secukinumab $300 \mathrm{mg}(\mathrm{N}=461)$ or secukinumab $150 \mathrm{mg}$ subcutaneously (with and without subcutaneous loading dose; $N=907)$ or placebo $(N=681)$. Once pooled, patients were stratified within each treatment arm by prior TNFi use (TNFi naive and TNF-IR) within each treatment arm. The FUTURE studies did not assess axial disease in PsA. Although the MAXIMISE study (NCT02721966) has recently demonstrated the safety and efficacy of secukinumab in clinical and imaging measures of axial PsA [20], we did not include this study in the current analysis because the entire population is TNFi naive.

All included studies were approved by each central institutional review board (IRB; FUTURE 2 approving board, Copernicus Group IRB; date of approval, January 17, 2013; Copernicus IRB Tracking number, NOV2 12439. 
FUTURE 3 approving board, Quorum IRB; date of approval, February 4, 2014. FUTURE 4 approving board, Chesapeake IRB; date of approval, December 12, 2014. FUTURE 5 approving board, Chesapeake IRB; date of approval, June 11, 2015). Approval was also obtained from the ethics review boards of each additional center that participated in the individual studies. All included studies were performed in accordance with the Helsinki Declaration of 1964 and its later amendments. Written informed consent was obtained from all participants before study inclusion.

\section{Outcomes and Assessments}

The efficacy of secukinumab at week 16 was evaluated according to the updated GRAPPAOMERACT PsA core domains using multiple instruments recorded in published clinical trials $[13,15]$.

\section{Musculoskeletal Disease Activity}

Arthritis, enthesitis, and dactylitis were assessed using at least 50\% improvement and complete resolution (100\%) in swollen joint count in 66 joints (SJC66), tender joint count in 68 joints (TJC68), Leeds Enthesitis Index (LEI) [21], and Leeds Dactylitis Index (LDI) [22].

\section{Skin and Nail Disease Activity}

Efficacy for psoriasis was assessed as achievement of at least 75\% improvement in the Psoriasis Area and Severity Index (PASI75) and complete resolution (PASI100), Investigator's Global Assessment modified 2011 scores of 0 or 1 (clear or almost clear), and 75\% improvement in the modified Nail Psoriasis Severity Index (mNAPSI75) [23].

\section{Symptoms and Life Impact}

Pain and patient global assessment were assessed by the mean change from baseline using a $100-\mathrm{mm}$ visual analog scale (VAS) and achievement of at least a 30\% improvement in pain and patient global assessment VAS scores. Physical function was assessed by the mean change from baseline in the Health Assessment Questionnaire-Disability Index (HAQ-DI; scale, 0-3) and by achievement of the minimal clinically important difference (MCID) of at least 0.35 in PsA [24, 25]. Health-related quality of life (HRQOL) was assessed by achievement of the MCID of at least 2.5 in the raw 36-Item Short Form Health Survey (SF-36) physical component summary (PCS) and SF-36 mental component summary (MCS) scores established and used in rheumatoid arthritis [26-29]. Fatigue was assessed using both the mean change from baseline in the Functional Assessment of Chronic Illness Therapy-Fatigue (FACIT-Fatigue; scale, 0-52) scores and achievement of the FACIT-Fatigue response, defined as a change from baseline of at least $4.0[30,31]$. Systemic inflammation was evaluated by the resolution of elevated C-reactive protein (CRP) levels at week 16 , defined as achievement of $10 \mathrm{mg} / \mathrm{L}$ or less among patients with CRP greater than $10 \mathrm{mg} / \mathrm{L}$ at baseline. Efficacy for overall activity impairment was assessed by achievement of MCID of absenteeism of at least $20 \%$, presenteeism of at least $20 \%$, work productivity loss of at least $15 \%$, and activity impairment of at least $20 \%$ in the respective Work Productivity and Activity Impairment Questionnaire: General Health (WPAI:GH) domains; these cutoffs were adapted from those thresholds reported in PsA using the WPAI Specific Health Questionnaire [32].

\section{Statistical Analysis}

Thresholds for meaningful improvement have been previously defined for several outcome measures. When an MCID was defined for an outcome measure, this threshold was used for this post hoc analysis. When an MCID was unknown, a threshold was selected that was judged to be meaningful on the basis of current measures in use (e.g., 50-75\% improvement) or least squares mean (LSM) change [13]. For musculoskeletal disease and skin disease activity, complete resolution (100\% improvement) was also assessed.

For each outcome, comparisons between secukinumab vs placebo were separately evaluated in the TNFi-naive and TNF-IR cohorts at week 16. Nonresponder imputation analysis for 
missing data was conducted for musculoskeletal disease activity, PASI, and activity impairment scores; patients with no baseline impairment by WPAI:GH were counted as nonresponders per convention as a conservative imputation approach. As-observed data were used in the analyses of other outcomes. Effect sizes for selected outcomes were presented as Cohen $d$ values, calculated as the mean change from baseline at week 16 divided by the standard deviation of the difference [33]. Effect size was interpreted here in either the positive or negative directions as 0.2 for small, 0.5 for moderate, and 0.8 for large [33]. All $P$ values were calculated for purposes of hypothesis generation, and no adjustment was made for multiple comparisons.

\section{RESULTS}

\section{Demographics and Clinical Characteristics}

This pooled analysis included 2049 participants (1436 TNFi naive and 613 TNF-IR) from the FUTURE 2, 3, 4, and 5 randomized controlled trials. Demographics and baseline disease characteristics of patients from each pooled trial are shown in Table $\mathrm{S} 1$ in the supplementary material. A mixed population of TNF-IR and TNFinaive patients were enrolled in each of the four studies; TNF-IR patients comprised $31.5 \%$, $29.1 \%$, and $30.0 \%$ of the secukinumab $300 \mathrm{mg}$, secukinumab $150 \mathrm{mg}$, and placebo arms, respectively. Baseline demographics and disease characteristics were broadly similar across all pooled arms (Table 1).

\section{Pooled Efficacy Across PsA Core Domains}

In the musculoskeletal disease activity domains (arthritis, enthesitis, dactylitis), TNFi-naive patients receiving secukinumab were numerically more likely to experience improvement than TNF-IR patients, although both groups receiving any dose of secukinumab were significantly more likely to achieve clinical endpoints than patients receiving placebo (Fig. 1a, b). Patients receiving secukinumab were more likely than those receiving placebo to experience improvement in swollen and tender joint counts (SJC66 and TJC68, at least 50\% improvement) and improvement in enthesitis and dactylitis (LEI and LDI at least 50\% improvement; all $P<0.05)$. Resolution of dactylitis and enthesitis was observed regardless of secukinumab dose and prior TNFi exposure (all $P<0.05$ ), although responses were numerically higher in TNFi-naive vs TNF-IR patients and inpatients receiving secukinumab $300 \mathrm{mg}$ vs those receiving secukinumab $150 \mathrm{mg}$. TNFinaive patients receiving secukinumab were more likely to experience resolution of both SJC66 and TJC68 than those receiving placebo (all $P<0.05$ ). However, among TNF-IR patients, only those receiving secukinumab $150 \mathrm{mg}$ were significantly more likely to experience SJC66 resolution than those receiving placebo $(P<$ $0.05)$, and no groups receiving secukinumab were significantly more likely to experience resolution of TJC68 than patients receiving placebo. This disparity between cohorts could be explained by worse arthritis among TNF-IR patients, as evidenced by slightly higher SJC66 and TJC68 scores at baseline vs TNFi-naive patients (Table 1 ), or possibly by a shift in disease phenotype within the TNF-IR population. Effect sizes for the musculoskeletal domains were largest in TNFi-naive patients treated with secukinumab $300 \mathrm{mg}$, and effect sizes were generally larger in TNFi-naive patients than in TNF-IR patients (Table 2).

In the skin disease activity domain, a significantly higher proportion of TNFi-naive and TNF-IR patients receiving secukinumab $300 \mathrm{mg}$ achieved PASI75 and PASI100 responses and Investigator's Global Assessment-modified 2011 scores of 0 or 1 at week 16 than patients receiving placebo (all $P<0.05$; Fig. 2). TNFinaive patients receiving either dose of secukinumab were numerically more likely to achieve PASI100 than TNF-IR patients in the corresponding treatment arm. Of patients with nail involvement at baseline, a significantly higher proportion of TNFi-naive and TNF-IR patients receiving both doses of secukinumab achieved an mNAPSI75 response compared with patients receiving placebo (all $P<0.05$ ), with a numerically greater proportion of TNFi-naive patients 
Table 1 Pooled baseline patient characteristics across FUTURE 2-5 trials, stratified by TNFi use

\begin{tabular}{|c|c|c|c|c|c|c|}
\hline \multirow{2}{*}{$\begin{array}{l}\text { Characteristic } \\
\text { TNFi status }\end{array}$} & \multicolumn{2}{|c|}{$\begin{array}{l}\text { Secukinumab } 300 \mathrm{mg} \\
(n=461)\end{array}$} & \multicolumn{2}{|c|}{$\begin{array}{l}\text { Secukinumab } 150 \mathrm{mg} \\
(n=907)\end{array}$} & \multicolumn{2}{|c|}{ Placebo $(n=681)$} \\
\hline & $\begin{array}{l}\text { Naive } \\
(n=316)\end{array}$ & $\begin{array}{l}\text { TNF-IR } \\
(n=145)\end{array}$ & $\begin{array}{l}\text { Naive } \\
(n=643)\end{array}$ & $\begin{array}{l}\text { TNF-IR } \\
(n=264)\end{array}$ & $\begin{array}{l}\text { Naive } \\
(n=477)\end{array}$ & $\begin{array}{l}\text { TNF-IR } \\
(n=204)\end{array}$ \\
\hline Age, mean $(S D)$, years & $48.2(12.4)$ & $49.4(13.6)$ & $47.9(12.2)$ & $50.9(11.7)$ & $48.5(12.2)$ & $51.2(12.4)$ \\
\hline Female, $n(\%)$ & $150(47.5)$ & $85(58.6)$ & $328(51.0)$ & $134(50.8)$ & $254(53.2)$ & $123(60.3)$ \\
\hline \multicolumn{7}{|l|}{ Race, $n(\%)$} \\
\hline White & $291(92.1)$ & $121(83.4)$ & $578(89.9)$ & $227(86.0)$ & $431(90.4)$ & $184(90.2)$ \\
\hline Asian & $17(5.4)$ & $12(8.3)$ & $42(6.5)$ & $23(8.7)$ & $30(6.3)$ & $8(3.9)$ \\
\hline Other* & $8(2.5)$ & $12(8.3)$ & $21(3.3)$ & $14(5.3)$ & $14(2.9)$ & $12(5.9)$ \\
\hline Unknown & 0 & 0 & $2(0.3)$ & 0 & $2(0.4)$ & 0 \\
\hline BMI, mean (SD), $\mathrm{kg} / \mathrm{m}^{2}$ & $28.7(5.7)$ & $30.7(6.3)$ & $29.2(6.2)$ & $30.9(6.5)$ & $29.1(6.0)$ & $30.1(6.4)$ \\
\hline Methotrexate use at baseline, $n(\%)$ & $160(50.6)$ & $67(46.2)$ & $329(51.2)$ & $116(43.9)$ & $259(54.3)$ & $80(39.2)$ \\
\hline TJC68, mean (SD) & $16.5(11.6)$ & $22.2(15.0)$ & $18.1(13.4)$ & $23.5(16.5)$ & $18.4(13.5)$ & $23.5(16.2)$ \\
\hline SJC66, mean (SD) & $8.5(5.7)$ & $10.5(8.3)$ & $9.7(7.7)$ & $12.2(10.2)$ & $9.9(7.9)$ & $11.2(10.3)$ \\
\hline Presence of enthesitis, $n$ (\%) & $195(61.7)$ & $89(61.4)$ & $382(59.4)$ & $187(70.8)$ & $291(61.0)$ & $140(68.6)$ \\
\hline Presence of dactylitis, $n$ (\%) & $117(37.0)$ & $57(39.3)$ & $236(36.7)$ & $93(35.2)$ & $167(35.0)$ & $64(31.4)$ \\
\hline DAS28-CRP score, mean (SD) & $4.40(1.02)$ & $4.92(0.97)$ & $4.49(1.03)$ & $5.03(1.09)$ & $4.54(1.03)$ & $4.81(1.13)$ \\
\hline DAS28-ESR score, mean (SD) & $4.84(1.17)$ & $5.35(1.19)$ & $4.89(1.16)$ & $5.45(1.25)$ & $4.98(1.20)$ & $5.29(1.23)$ \\
\hline PASDAS score $(0-10)$, mean (SD) & $5.65(1.03)$ & $6.03(0.95)$ & $5.73(1.05)$ & $6.26(1.02)$ & $5.72(1.07)$ & $6.00(1.03)$ \\
\hline PASI score $(0-72)$, mean $(S D)$ & $\begin{array}{l}11.12 \\
(9.01)\end{array}$ & $11.28(8.45)$ & $\begin{array}{l}11.84 \\
(11.17)\end{array}$ & $\begin{array}{l}12.54 \\
\quad(10.11)\end{array}$ & $\begin{array}{l}10.86 \\
(9.38)\end{array}$ & $11.31(9.23)$ \\
\hline $\begin{array}{l}\text { PsA pain score (VAS 0-100), mean } \\
\text { (SD) }\end{array}$ & $52.2(23.2)$ & $59.4(23.1)$ & $53.2(23.0)$ & $61.1(20.7)$ & $52.4(23.2)$ & $58.4(23.5)$ \\
\hline $\mathrm{CRP}>10 \mathrm{mg} / \mathrm{L}, n(\%)$ & $70(22.2)$ & $45(31.0)$ & $134(20.8)$ & $85(32.2)$ & $115(24.1)$ & $52(25.5)$ \\
\hline HAQ-DI score (0-3), mean (SD) & $1.12(0.62)$ & $1.34(0.65)$ & $1.12(0.63)$ & $1.43(0.63)$ & $1.18(0.61)$ & $1.35(0.66)$ \\
\hline $\begin{array}{l}\text { SF-36 PCS score }(0-100) \text {, mean } \\
\text { (SD) }\end{array}$ & $\begin{array}{l}38.64 \\
(8.10)\end{array}$ & $35.66(8.05)$ & $\begin{array}{l}38.49 \\
(8.19)\end{array}$ & $33.82(8.29)$ & $\begin{array}{l}37.85 \\
(8.30)\end{array}$ & $34.42(8.23)$ \\
\hline $\begin{array}{l}\text { SF-36 MCS score }(0-100) \text {, mean } \\
\text { (SD) }\end{array}$ & $\begin{array}{l}44.19 \\
\quad(11.58)\end{array}$ & $\begin{array}{l}43.80 \\
\quad(12.01)\end{array}$ & $\begin{array}{l}43.69 \\
(10.98)\end{array}$ & $\begin{array}{l}42.31 \\
\quad(10.97)\end{array}$ & $\begin{array}{l}44.50 \\
\quad(10.67)\end{array}$ & $\begin{array}{l}42.30 \\
\quad(12.19)\end{array}$ \\
\hline $\begin{array}{l}\text { FACIT-Fatigue score }(0-52) \text {, mean } \\
(\mathrm{SD})\end{array}$ & $31.3(11.4)$ & $27.1(12.1)$ & $30.8(11.2)$ & $25.0(11.8)$ & $31.1(10.5)$ & $25.4(11.3)$ \\
\hline $\begin{array}{l}\text { Patient global assessment score (VAS } \\
0-100) \text {, mean (SD) }\end{array}$ & $55.3(21.3)$ & $62.9(21.1)$ & $54.8(22.2)$ & $64.0(20.8)$ & $55.2(20.9)$ & $60.1(21.6)$ \\
\hline
\end{tabular}


Table 1 continued

\begin{tabular}{|c|c|c|c|c|c|c|}
\hline \multirow{2}{*}{$\begin{array}{l}\text { Characteristic } \\
\text { TNFi status }\end{array}$} & \multicolumn{2}{|c|}{$\begin{array}{l}\text { Secukinumab } 300 \mathrm{mg} \\
(n=461)\end{array}$} & \multicolumn{2}{|c|}{$\begin{array}{l}\text { Secukinumab } 150 \mathrm{mg} \\
(n=907)\end{array}$} & \multicolumn{2}{|c|}{ Placebo $(n=681)$} \\
\hline & $\begin{array}{l}\text { Naive } \\
(n=316)\end{array}$ & $\begin{array}{l}\text { TNF-IR } \\
(n=145)\end{array}$ & $\begin{array}{l}\text { Naive } \\
(n=643)\end{array}$ & $\begin{array}{l}\text { TNF-IR } \\
(n=264)\end{array}$ & $\begin{array}{l}\text { Naive } \\
(n=477)\end{array}$ & $\begin{array}{l}\text { TNF-IR } \\
(n=204)\end{array}$ \\
\hline \multicolumn{7}{|l|}{ WPAI:GH score, mean (SD) $[n]$} \\
\hline Work time missed (absenteeism) & $\begin{array}{c}8.5(19.2) \\
{[132]}\end{array}$ & $\begin{array}{c}11.2(22.8) \\
{[45]}\end{array}$ & $\begin{array}{c}12.3(25.5) \\
{[253]}\end{array}$ & $\begin{array}{l}19.0(27.6) \\
{[86]}\end{array}$ & $\begin{array}{c}10.3(21.4) \\
{[183]}\end{array}$ & $\begin{array}{l}14.0(25.4) \\
{[62]}\end{array}$ \\
\hline $\begin{array}{l}\text { Impairment while working } \\
\text { (presenteeism) }\end{array}$ & $\begin{array}{c}35.3(25.0) \\
{[131]}\end{array}$ & $\begin{array}{l}37.5(25.5) \\
{[44]}\end{array}$ & $\begin{array}{c}34.1(25.5) \\
{[242]}\end{array}$ & $\begin{array}{l}47.4(25.2) \\
\quad[82]\end{array}$ & $\begin{array}{c}37.8(22.9) \\
{[180]}\end{array}$ & $\begin{array}{l}44.2(24.2) \\
{[60]}\end{array}$ \\
\hline Overall work impairment & $\begin{array}{c}38.7(27.4) \\
{[130]}\end{array}$ & $\begin{array}{l}41.2(27.5) \\
{[44]}\end{array}$ & $\begin{array}{c}38.0(27.9) \\
{[242]}\end{array}$ & $\begin{array}{l}54.1(26.5) \\
{[82]}\end{array}$ & $\begin{array}{c}42.0(24.6) \\
{[179]}\end{array}$ & $\begin{array}{c}47.9(26.8) \\
{[59]}\end{array}$ \\
\hline Activity impairment & $\begin{array}{c}44.9(25.2) \\
{[202]}\end{array}$ & $\begin{array}{l}52.2(27.1) \\
{[87]}\end{array}$ & $\begin{array}{c}44.9(24.9) \\
{[431]}\end{array}$ & $\begin{array}{c}56.8(22.9) \\
{[157]}\end{array}$ & $\begin{array}{c}47.0(23.8) \\
{[315]}\end{array}$ & $\begin{array}{c}53.7(25.3) \\
{[119]}\end{array}$ \\
\hline
\end{tabular}

$B M I$ body mass index, CRP C-reactive protein, $D A S 28$ disease activity score using 28 joints, ESR erythrocyte sedimentation rate, FACIT-Fatigue Functional Assessment of Chronic Illness Therapy-Fatigue, HAQ-DI Health Assessment Questionnaire Disability Index, $M C S$ mental component summary, PASDAS Psoriatic Arthritis Disease Activity Score, PASI Psoriasis Area and Severity Index, $P C S$ physical component summary, $P s A$ psoriatic arthritis, $S F$-36 36-Item Short Form Health Survey, SJC swollen joint count, TJC tender joint count, TNFi tumor necrosis factor inhibitor, TNF-IR tumor necrosis factor inhibitor inadequate responder, $V A S$ visual analog scale, WPAI:GH Work Productivity and Activity Impairment Questionnaire: General Health

*Includes Black or African American, American Indian or Alaskan Native, Native Hawaiian or other Pacific Islander, and other

achieving responses vs TNF-IR patients. Treatment effect sizes in psoriasis were large and highest for secukinumab $300 \mathrm{mg}$ vs $150 \mathrm{mg}$, irrespective of previous TNFi experience (Table 2). The same effect was seen for Dermatology Quality of Life Index. Treatment effect sizes were moderate in nail psoriasis and similar between secukinumab doses and between TNFinaive and TNF-IR cohorts.

Significant improvements were observed with both secukinumab doses vs placebo at week 16 in the pain and physical function domains, as determined by at least a 30\% improvement in the PsA pain VAS and at least a 0.35 -point improvement in HAQ-DI, respectively, regardless of prior TNFi use (all $P<0.05$; Fig. 3a). Further, TNFi-naive patients receiving secukinumab were generally numerically more likely to experience clinical response than TNFIR patients. Patients receiving secukinumab also experienced significant benefits in the HRQOL (Fig. 3b) and systemic inflammation domains (Fig. 3c). Patients treated with both doses of secukinumab were significantly more likely to achieve at least 2.5-point improvements in both the SF-36 PCS and SF-36 MCS at week 16 compared with those treated with placebo. For SF-36 PCS, patients receiving secukinumab $300 \mathrm{mg}$ were numerically more likely to achieve clinical response compared with those receiving secukinumab $150 \mathrm{mg}$. The observation that TNFinaive patients receiving any dose of secukinumab experienced clinical responses slightly more frequently than those who were TNF-IR is also reflected in Cohen $d$ effect sizes of treatment (Table 2). Effect sizes for physical function (HAQ-DI) were large in the secukinumab 300-mg group regardless of previous TNFi use and in TNFi-naive patients treated with secukinumab $150 \mathrm{mg}$; the HAQ-DI effect size was 


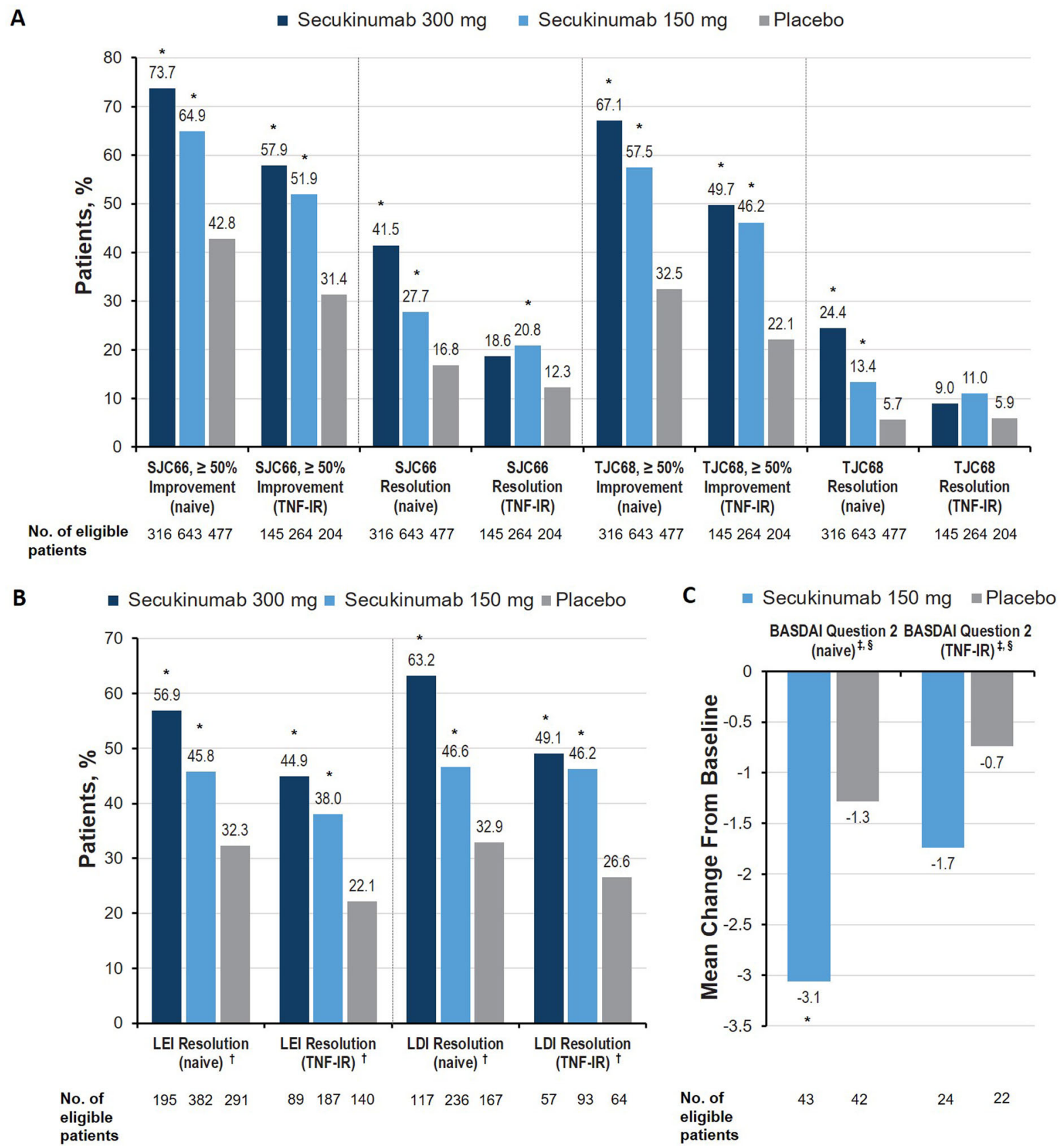

Fig. 1 Percentage of patients with improvement across the musculoskeletal disease activity domain in terms of A arthritis, B enthesitis and dactylitis, $\mathbf{C}$ spinal and hip pain assessed using BASDAI item \#2 in patients with PsA at week 16 in the FUTURE 2-5 trials. BASDAI Bath Ankylosing Spondylitis Disease Activity Index, LDI Leeds

moderate in TNF-IR patients treated with secukinumab $150 \mathrm{mg}$. This trend was similar for
Dactylitis Index, LEI Leeds Enthesitis Index, LSM least squares mean, PsA psoriatic arthritis, SJC swollen joint count, TJC tender joint count, TNF-IR tumor necrosis factor inhibitor inadequate responder. ${ }^{\dagger}$ Among patients with enthesitis or dactylitis at baseline only. ${ }^{*} P<0.05$ vs placebo

HRQOL as measured by the SF-36 PCS. SF-36 MCS effect sizes were small for secukinumab- 
Table 2 Cohen $d$ effect sizes for selected outcomes in TNFi-naive and TNF-IR patients vs placebo in the FUTURE 2-5 trials

\begin{tabular}{|c|c|c|c|c|c|c|}
\hline \multirow{2}{*}{$\begin{array}{l}\text { Measure, Cohen } d \text { of mean } \\
\text { change from baseline } \\
\text { TNFi status }\end{array}$} & \multicolumn{2}{|c|}{$\begin{array}{l}\text { Secukinumab } 300 \mathrm{mg} \\
(n=461)\end{array}$} & \multicolumn{2}{|c|}{$\begin{array}{l}\text { Secukinumab } 150 \mathrm{mg} \\
(n=907)\end{array}$} & \multicolumn{2}{|c|}{ Placebo $(n=681)$} \\
\hline & $\begin{array}{l}\text { Naive } \\
(n=316)\end{array}$ & $\begin{array}{l}\text { TNF-IR } \\
(n=145)\end{array}$ & $\begin{array}{l}\text { Naive } \\
(n=643)\end{array}$ & $\begin{array}{l}\text { TNF-IR } \\
(n=264)\end{array}$ & $\begin{array}{l}\text { Naive } \\
(n=477)\end{array}$ & $\begin{array}{l}\text { TNF-IR } \\
(n=204)\end{array}$ \\
\hline \multicolumn{7}{|l|}{ Musculoskeletal domain } \\
\hline SJC66 & -0.83 & -0.67 & -0.78 & -0.48 & -0.49 & -0.13 \\
\hline TJC68 & -0.84 & -0.60 & -0.70 & -0.48 & -0.33 & -0.10 \\
\hline LDI & -0.30 & -0.19 & -0.23 & -0.15 & -0.11 & -0.08 \\
\hline LEI & -0.59 & -0.44 & -0.44 & -0.39 & -0.30 & -0.01 \\
\hline \multicolumn{7}{|l|}{ Skin } \\
\hline PASI score & -1.11 & -1.16 & -0.90 & -0.95 & -0.31 & -0.01 \\
\hline IGA mod 2011 score & -1.73 & -1.75 & -1.39 & -1.05 & -0.45 & -0.39 \\
\hline mNAPSI & -0.68 & -0.63 & -0.63 & -0.52 & -0.19 & -0.10 \\
\hline \multicolumn{7}{|l|}{ Pain and physical function } \\
\hline PsA pain & -0.83 & -0.56 & -0.73 & -0.53 & -0.29 & -0.10 \\
\hline \multicolumn{7}{|l|}{ HRQOL } \\
\hline HAQ-DI & -0.97 & -0.75 & -0.81 & -0.64 & -0.48 & -0.19 \\
\hline SF-36 PCS & 0.94 & 0.76 & 0.78 & 0.58 & 0.37 & 0.23 \\
\hline SF-36 MCS & 0.42 & 0.32 & 0.44 & 0.30 & 0.16 & 0.11 \\
\hline PsAQOL & -0.75 & -0.54 & -0.68 & -0.49 & -0.27 & -0.12 \\
\hline DLQI & -0.92 & -0.95 & -1.01 & -1.00 & -0.32 & -0.35 \\
\hline \multicolumn{7}{|l|}{ Systemic inflammation } \\
\hline CRP & -0.38 & -0.41 & -0.30 & -0.33 & -0.05 & -0.02 \\
\hline ESR & -0.69 & -0.49 & -0.52 & -0.44 & -0.24 & 0.03 \\
\hline \multicolumn{7}{|l|}{ Fatigue } \\
\hline FACIT-Fatigue & 0.66 & 0.64 & 0.63 & 0.47 & 0.25 & 0.12 \\
\hline \multicolumn{7}{|l|}{ Patient global } \\
\hline Patient global assessment & -0.77 & -0.68 & -0.61 & -0.63 & -0.33 & -0.17 \\
\hline Participation & & & & & & \\
\hline
\end{tabular}


Table 2 continued

\begin{tabular}{|c|c|c|c|c|c|c|}
\hline \multirow{2}{*}{$\begin{array}{l}\text { Measure, Cohen } d \text { of mean } \\
\text { change from baseline } \\
\text { TNFi status }\end{array}$} & \multicolumn{2}{|c|}{$\begin{array}{l}\text { Secukinumab } 300 \mathrm{mg} \\
(n=461)\end{array}$} & \multicolumn{2}{|c|}{$\begin{array}{l}\text { Secukinumab } 150 \mathrm{mg} \\
(n=907)\end{array}$} & \multicolumn{2}{|c|}{ Placebo $(n=681)$} \\
\hline & $\begin{array}{l}\text { Naive } \\
(n=316)\end{array}$ & $\begin{array}{l}\text { TNF-IR } \\
(n=145)\end{array}$ & $\begin{array}{l}\text { Naive } \\
(n=643)\end{array}$ & $\begin{array}{l}\text { TNF-IR } \\
(n=264)\end{array}$ & $\begin{array}{l}\text { Naive } \\
(n=477)\end{array}$ & $\begin{array}{l}\text { TNF-IR } \\
(n=204)\end{array}$ \\
\hline WPAI:GH & -0.58 & -0.49 & -0.48 & -0.40 & -0.25 & $<0.01$ \\
\hline
\end{tabular}

Effect size interpretation is subjective; generally, a magnitude in either the positive or negative direction was considered as 0.2 for small, 0.5 for moderate, 0.8 for large [33]

CRP C-reactive protein, DLQI Dermatology Life Quality Index, ESR erythrocyte sedimentation rate, FACIT-Fatigue Functional Assessment of Chronic Illness Therapy-Fatigue, HAQ-DI Health Assessment Questionnaire Disability Index, HRQOL health-related quality of life, IGA mod 2011 Investigator's Global Assessment modified 2011, LDI Leeds Dactylitis Index, LEI Leeds Enthesitis Index, MCS mental component summary, mNAPSI modified Nail Psoriasis Severity Index, $P A S I$ Psoriasis Area and Severity Index, PCS physical component summary, Ps $A$ psoriatic arthritis, $P s A Q O L$ Psoriatic Arthritis Quality of Life, SF-36 36-item Short Form Health Survey, SJC swollen joint count, TJC tender joint count, TNFi tumor necrosis factor inhibitor, TNF-IR tumor necrosis factor inhibitor inadequate responder, $V A S$ visual analog scale, WPAI:GH Work Productivity and Activity Impairment Questionnaire: General Health

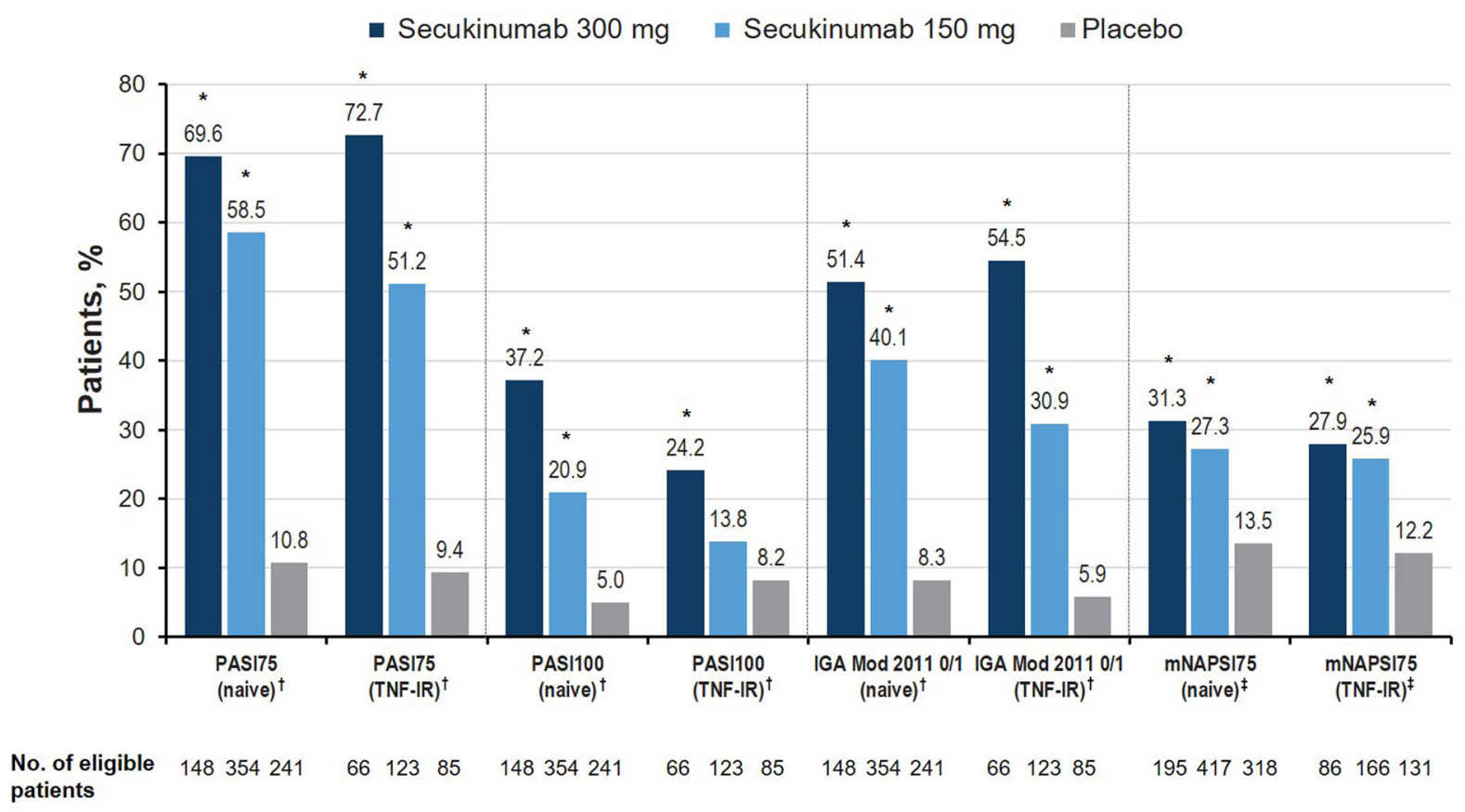

Fig. 2 Percentage of patients with meaningful improvement in the skin disease activity domain at week 16 in the FUTURE 2-5 trials. IGA mod 2011 Investigator's Global Assessment modified 2011, LSM least squares mean, mNAPSI modified Nail Psoriasis Severity Index, PASI

treated patients, although the TNFi-naive cohort had the highest effect sizes. For the PsA-
Psoriasis Area and Severity Index, TNF-IR tumor necrosis factor inhibitor inadequate responder. ${ }^{\dagger}$ Among patients with affected body surface area of at least 3\% at baseline. $\ddagger$ Among patients with nail involvement at baseline. ${ }^{*} P<0.05$ vs placebo

specific HRQOL (PsAQOL), the effect sizes were moderate for all groups, except for TNF-IR 
A - Secukinumab $300 \mathrm{mg}$ ॥ Secukinumab $150 \mathrm{mg}$ —Placebo

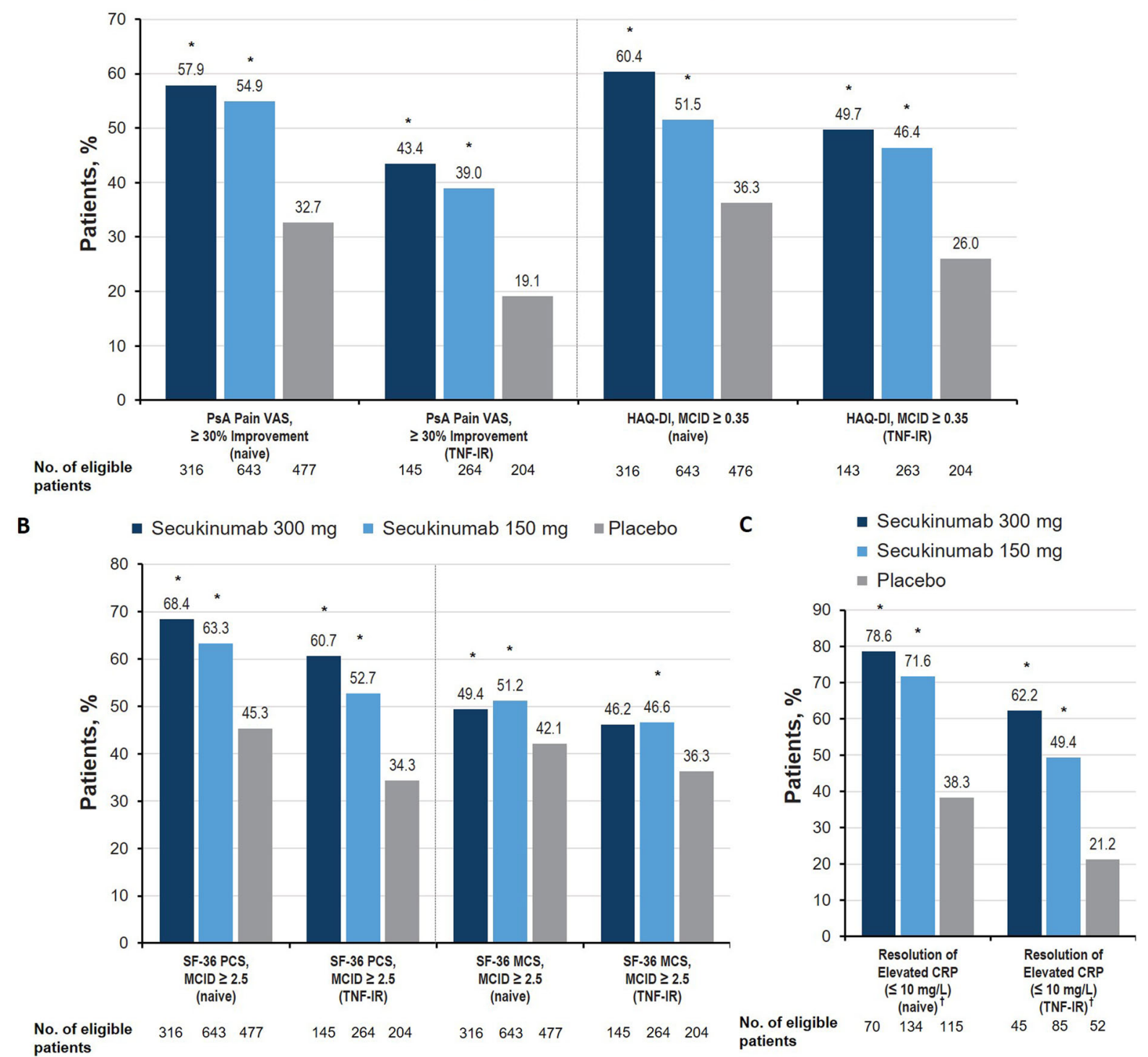

Fig. 3 Percentage of patients with meaningful improvement in the $\mathbf{a}$ pain and physical function, $\mathbf{b}$ health-related quality of life, and $\mathbf{c}$ systemic inflammation domains at week 16 in the FUTURE 2-5 trials. CRP C-reactive protein, LSM least squares mean, MCID minimal clinically important difference, MCS mental component summary,

patients treated with secukinumab $150 \mathrm{mg}$, in whom the effect size was small.

Of patients with elevated CRP at baseline (CRP greater than $10 \mathrm{mg} / \mathrm{L}$ ), a significantly higher proportion of those receiving secukinumab experienced improvement in the systemic inflammation domain (CRP reduction
PCS physical component summary, PsA psoriatic arthritis, SF-36 36-item Short Form Health Survey, TNF-IR tumor necrosis factor inhibitor inadequate responder, VAS visual analog scale. ${ }^{\dagger}$ Among patients with CRP greater than $10 \mathrm{mg} / \mathrm{L}$ at baseline. ${ }^{*} P<0.05$ vs placebo

less than $10 \mathrm{mg} / \mathrm{L})$ compared with patients receiving placebo, regardless of prior TNFi use (all $P<0.05$ ). These responses were reflected in CRP-level effect sizes, which were similar for secukinumab-treated patients regardless of dose and TNFi status and were greater than those in patients receiving placebo (Table 2). 
A - Secukinumab $300 \mathrm{mg}$ @ Secukinumab $150 \mathrm{mg} \backsim$ Placebo

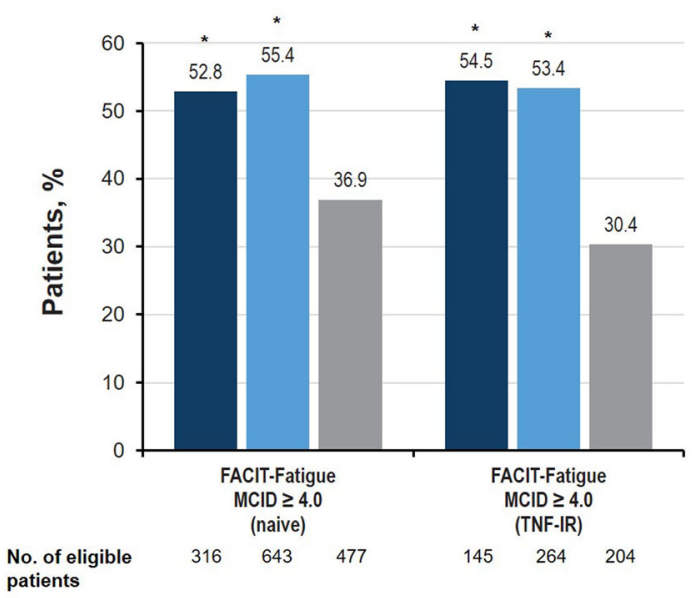

Fig. 4 Percentage of patients with meaningful improvement across a fatigue and $\mathbf{b}$ patient global assessment domains in patients with PsA at week 16 in the FUTURE 2-5 trials. FACIT-Fatigue Functional
B - Secukinumab $300 \mathrm{mg}$ = Secukinumab $150 \mathrm{mg}$ @ Placebo

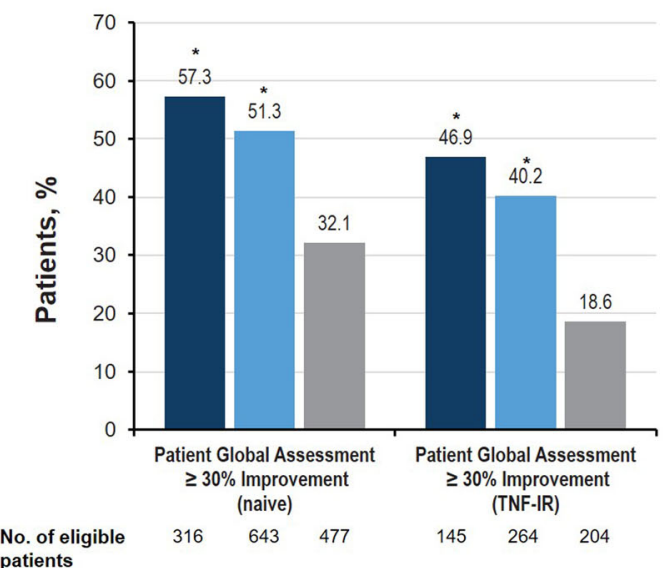

Assessment of Chronic Illness Therapy-Fatigue, MCID minimal clinically important difference, PsA psoriatic arthritis, TNF-IR tumor necrosis factor inhibitor inadequate responder. ${ }^{*} P<0.05$ vs placebo

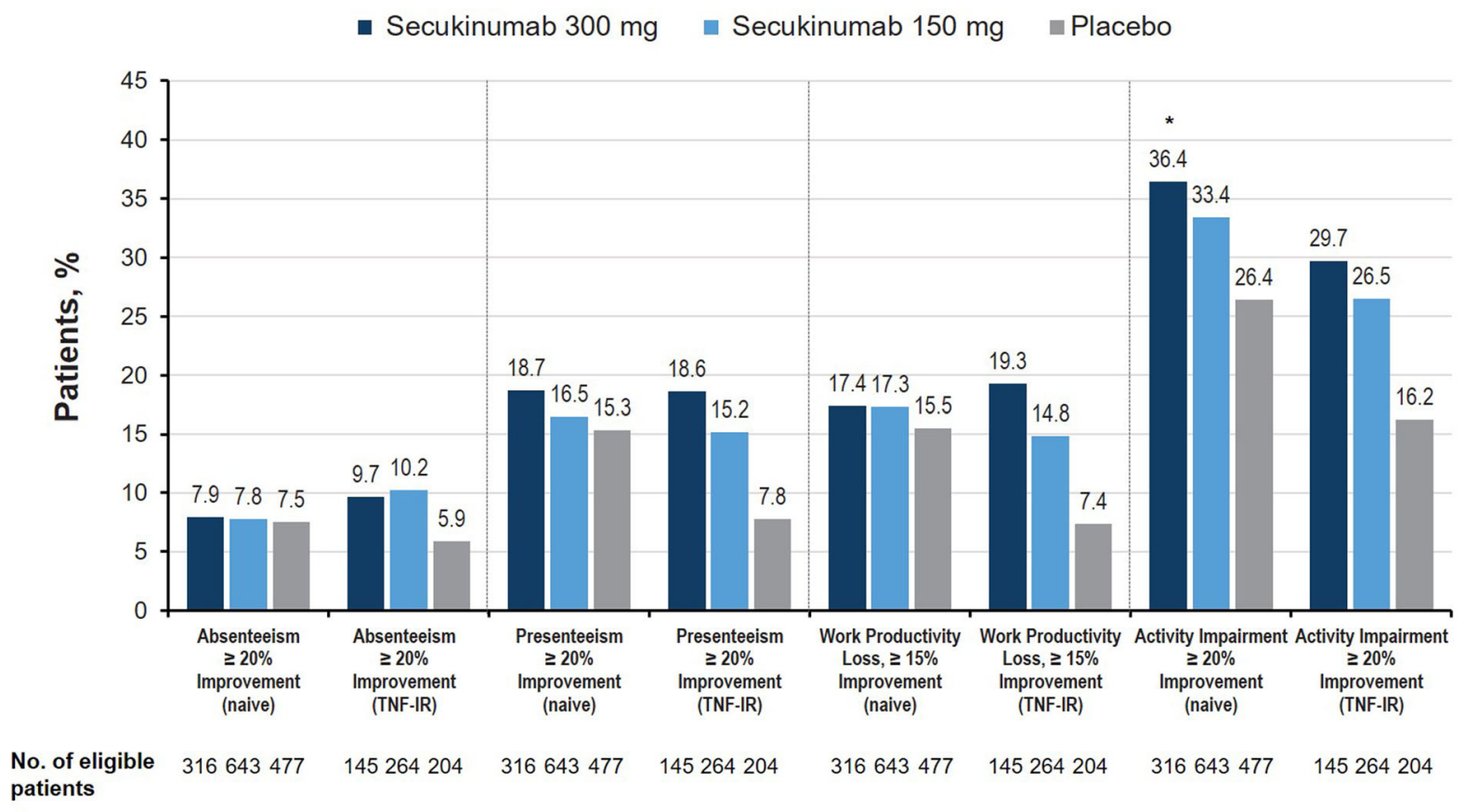

Fig. 5 Percentage of patients with meaningful improvement in the participation domain at week 16 in the FUTURE 2-5 trials (nonresponder imputation). For all analyses, patients without baseline impairment in WPAI domains were characterized as nonresponders. TNF-IR tumor necrosis factor inhibitor inadequate responder, WPAI Work Productivity and Activity Impairment questionnaire. ${ }^{*} P<0.05$ vs placebo 
Both doses of secukinumab showed significant improvements at week 16 in the fatigue and patient global assessment domain vs placebo, regardless of prior TNFi use $(P<0.05$ vs placebo; Fig. 4a, b). Achievement of responses was numerically more likely for patients receiving secukinumab $300 \mathrm{mg}$ than those receiving secukinumab $150 \mathrm{mg}$, and TNFi-naive patients showed numerically greater improvements with secukinumab than those who were TNF-IR. For fatigue, effect sizes for secukinumab-treated patients were moderate, although the effect size was small for TNF-IR patients treated with secukinumab $150 \mathrm{mg}$ (Table 2).

Treatment with secukinumab also resulted in significant improvement in the GRAPPA middle circle domain of work participation for TNFinaive and TNF-IR cohorts. All groups receiving secukinumab showed significant improvements in impairment of participation, as determined by proportion of patients achieving at least $20 \%$ improvement in the WPAI:GH activity impairment domain at week 16 compared with those receiving placebo $(P<0.05$; Fig. 5). TNF-IR patients receiving any dose of secukinumab were also more likely than those receiving placebo to achieve at least $20 \%$ improvement in presenteeism and at least $15 \%$ improvement in work productivity loss at week $16 \quad(P<0.05$; Fig. 5). Effect sizes for activity impairment were moderate in TNFi-naive patients treated with secukinumab $300 \mathrm{mg}$ and small in those treated with secukinumab $150 \mathrm{mg}$; effect sizes were small in all TNF-IR patients receiving either dose of secukinumab.

\section{DISCUSSION}

In this retrospective, post hoc analysis comparing the efficacy of secukinumab to that of placebo in TNF-IR and TNFi-naive patients across individual GRAPPA domains, secukinumab was found to benefit patients across all domains, regardless of prior TNFi exposure. To ascertain the breadth of effects of secukinumab on individual disease domains, we deconstructed the composite measures used in the FUTURE 2-5 trials and assessed discrete outcomes. This is the first study to independently assess how TNF-IR and TNFi-naive patients respond to a biologic across all GRAPPA domains.

Generally, patients receiving secukinumab $300 \mathrm{mg}$ achieved clinical responses more frequently than those receiving secukinumab $150 \mathrm{mg}$. TNFi-naive patients receiving any dose of secukinumab tended to demonstrate improvements to a greater extent than those who were TNF-IR, except for psoriasis and related skin and nail outcomes, where improvements were generally similar between cohorts. For example, although improvement was noted in the musculoskeletal and skin domains for secukinumab-treated patients regardless of previous TNFi use, patients who were TNF-IR were numerically less likely to achieve complete resolution of joint tenderness and swelling or resolution of psoriasis as measured by PASI100. Effect sizes were generally highest for TNFinaive patients treated with secukinumab $300 \mathrm{mg}$ and lowest for TNF-IR patients treated with secukinumab $150 \mathrm{mg}$, although effect sizes for all secukinumab-treated patient groups were consistently higher than those receiving placebo. On the basis of this analysis, the higher secukinumab dose (300 mg) should be considered for patients who have previously experienced TNFi failure.

Longitudinal analyses of patients with PsA, together with the use of updated GRAPPA domains in clinical trials, have highlighted the need to treat the combination of disease symptoms uniquely affecting patients with PsA. TNFis have traditionally been used as first-line biologic treatments for PsA; however, in clinical trials, about $40 \%$ of patients with PsA fail to achieve American College of Rheumatology (ACR)20 responses [34, 35]. TNFi cycling is common in patients who experience inadequate response, adverse events, or tolerability issues [34, 35]. In an observational study from the Corrona registry, patients with previous TNFi exposure were more likely to discontinue or switch their current TNFi than patients without previous TNFi experience, and time to discontinuation was shorter [36]. A recent study of patients from the Swiss Registry for Clinical Quality Management in Rheumatology found that patients with PsA who switched from a 
TNFi to a biologic with a different mechanism of action experienced improved drug retention compared with those who switched to another TNFi [37]. Results from a randomized, doubleblind, phase 3 comparison of secukinumab vs the TNFi adalimumab (EXCEED; NCT02745080) suggested that secukinumab was at least as effective as adalimumab at improving joint symptoms for patients with PsA while offering increased benefit in the skin domain [38]. These results were consistent with observations from the SPIRIT-H2H study comparing ixekizumab with adalimumab in patients with PsA [39], adding evidence that biologics targeting IL-17A may offer benefit over TNFis at improving skin and joint manifestations of PsA.

Although psoriatic disease refractory to TNFi treatment is seen as more difficult to treat [5], biologics targeting PsA-specific inflammatory cytokines may offer clinical benefit and should be considered as first-line agents. The observed improvements with secukinumab treatment in TNF-IR and especially in TNFi-naive patient populations across all GRAPPA domains strengthen the evidence supporting secukinumab use as a first-line PsA therapy. Analyses of clinical practice data have shown that secukinumab is commonly prescribed to biologic-experienced patients [40]. Secukinumab has demonstrated superior persistence compared with other biologics in these patient cohorts [41] as well as compared with adalimumab in the EXCEED trial [38].

A few limitations should be considered when interpreting the findings from this analysis. As this is a post hoc analysis, data from the FUTURE studies were pooled from trials conducted at different times and places, with different patient populations. Data from these studies were pooled at the end of the doubleblind, placebo-controlled phase after 16 weeks of treatment. This short time frame may have been insufficient to allow the TNF-IR patients to achieve responses; however, we still noted that secukinumab-treated TNF-IR patients achieved meaningful clinical responses. Axial disease was not measured in the FUTURE 2-5 trials, and no data were available on axial disease among patients with PsA treated with secukinumab at the time of this analysis. No direct comparisons exist for the effect of secukinumab on axial disease in TNFi-naive and TNF-IR patients with PsA; however, results from the MAXIMISE trial have since demonstrated the efficacy of secukinumab on axial manifestations among biologic-naive patients with PsA [20]. Finally, no adjustments for multiple comparisons were made to the analyses presented here.

\section{CONCLUSIONS}

In this post hoc analysis, secukinumab demonstrated efficacy compared with placebo across GRAPPA-OMERACT PsA core domains in the phase 3 clinical trials program regardless of prior TNFi use, although TNFi-naive patients generally experienced the greatest benefits. These results further support the value of secukinumab in improving the heterogeneous PsA disease spectrum, including HRQOL measures, in both TNFi-naive and TNF-IR patients. On the basis of numerically greater responses in TNFi-naive compared with TNF-IR patients, secukinumab may be a preferred agent for the initial treatment of PsA.

\section{ACKNOWLEDGEMENTS}

Funding. This study and the Rapid Service Fee was funded by Novartis Pharmaceuticals Corporation, East Hanover, NJ, USA. Support for third-party writing assistance for this manuscript was provided by Novartis.

Medical Writing and Editorial Assistance. The authors thank Richard Karpowicz, $\mathrm{PhD}$, of Health Interactions, Inc, Hamilton, NJ, USA, for providing medical writing support/editorial support, which was funded by Novartis Pharmaceuticals Corporation, East Hanover, NJ, in accordance with Good Publication Practice (GPP3) guidelines (http://www.ismpp.org/gpp3)

Authorship. All named authors meet the International Committee of Medical Journal Editors criteria for authorship for this article, take responsibility for the integrity of the work 
as a whole, and have given their approval for this version to be published.

Authorship Contributions. A-MO, MEH, DDG, YYL, SS, WT, MV, OC, XM, PJ Mease contributed to the design of this study, data analysis and interpretation, and drafting of the manuscript.

Prior Presentation. A portion of these results were presented at the European League Against Rheumatism Annual European Congress of Rheumatology; June 12-15, 2019; Madrid, Spain.

Disclosures. A.-M. Orbai: received research grant support from AbbVie, Celgene, Horizon, Janssen, Lilly, and Novartis; received consulting fees from Janssen, Lilly, Novartis, Pfizer, and UCB; is a Jerome L. Greene Foundation Scholar; is supported in part by a research grant from the National Institute of Arthritis and Musculoskeletal and Skin Diseases of the National Institutes of Health under award number P30AR070254 (Core B), a Rheumatology Research Foundation Scientist Development Award, and a Staurulakis Family Discovery Award; is an Assistant Professor in the Department of Medicine, Division of Rheumatology, at the Johns Hopkins University School of Medicine; engaged in this research as an author and not in her capacity as a Johns Hopkins faculty member and was not compensated in income for being an author on this manuscript; and received no compensation from the National Institutes of Health for the development of this manuscript. M.E. Husni: received research grant support from Pfizer and consulting fees from AbbVie, Amgen, Gilead, Janssen, Lilly, Novartis, Pfizer, Regeneron, and UCB. D.D. Gladman: received research grant support from AbbVie, Amgen, Celgene, Lilly, Novartis, Pfizer, and UCB and consulting fees from AbbVie, Amgen, Bristol Myers Squibb, Celgene, Galapagos, Gilead, Janssen, Lilly, Novartis, Pfizer, and UCB. Y.Y. Leung: received speakers bureau fees from Janssen, AbbVie, Lilly, and Novartis and is a member of the editorial board of BMC Rheumatology. S. Siebert: received research grant support from Bristol Myers Squibb,
Boehringer Ingelheim, Celgene, Janssen, Novartis, Pfizer, GSK, and UCB; received consulting fees from AbbVie, Boehringer Ingelheim, Janssen, Novartis, Pfizer, and UCB; and received speakers bureau fees from AbbVie, Janssen, MSD, Novartis, Pfizer, and UCB. W. Tillett: received research grant support from AbbVie, Celgene, and Lilly; received consulting fees from AbbVie, Celgene, Lilly, Novartis, UCB, and Pfizer; and received speakers bureau fees from AbbVie, Celgene, Lilly, Janssen, Novartis, Pfizer, and UCB. M. Vis: received research grant support from Novartis. O. Chambenoit and X. Meng: employees and stockholders of Novartis. P.J. Mease: received research grants from AbbVie, Amgen, Bristol Myers Squibb, Eli Lilly, Galapagos, Janssen, Novartis, Pfizer, Sun Pharma, and UCB; received consulting fees from AbbVie, Amgen, Boehringer Ingelheim, Bristol Myers Squibb, Eli Lilly, Galapagos, Gilead, GlaxoSmithKline, Janssen, Novartis, Pfizer, Sun Pharma, and UCB; and received speakers bureau fees from AbbVie, Amgen, Eli Lilly, Janssen, Novartis, Pfizer, and UCB.

Compliance with Ethics Guidelines. All included studies were approved by each central institutional review board (IRB; FUTURE 2 approving board, Copernicus Group IRB; date of approval, January 17, 2013; Copernicus IRB Tracking number, NOV2 12 439. FUTURE 3 approving board, Quorum IRB; date of approval, February 4, 2014. FUTURE 4 approving board, Chesapeake IRB; date of approval, December 12, 2014. FUTURE 5 approving board, Chesapeake IRB; date of approval, June 11, 2015). Approval was also obtained from the ethics review boards of each additional center that participated in the individual studies. All included studies were performed in accordance with the Helsinki Declaration of 1964 and its later amendments. Written informed consent was obtained from all participants before study inclusion.

Data Availability. All data generated or analyzed during this study are included in this published article.

Open Access. This article is licensed under a Creative Commons Attribution- 
NonCommercial 4.0 International License, which permits any non-commercial use, sharing, adaptation, distribution and reproduction in any medium or format, as long as you give appropriate credit to the original author(s) and the source, provide a link to the Creative Commons licence, and indicate if changes were made. The images or other third party material in this article are included in the article's Creative Commons licence, unless indicated otherwise in a credit line to the material. If material is not included in the article's Creative Commons licence and your intended use is not permitted by statutory regulation or exceeds the permitted use, you will need to obtain permission directly from the copyright holder. To view a copy of this licence, visit http:// creativecommons.org/licenses/by-nc/4.0/.

\section{REFERENCES}

1. Alinaghi F, Calov M, Kristensen LE, et al. Prevalence of psoriatic arthritis in patients with psoriasis: a systematic review and meta-analysis of observational and clinical studies. J Am Acad Dermatol. 2019;80(1):251-65.e19.

2. Ritchlin CT, Colbert RA, Gladman DD. Psoriatic arthritis. N Engl J Med. 2017;376(10):957-70.

3. Gladman DD, Investigators AS, Sampalis JS, Illouz $\mathrm{O}$, Guerette B. Responses to adalimumab in patients with active psoriatic arthritis who have not adequately responded to prior therapy: effectiveness and safety results from an open-label study. J Rheumatol. 2010;37(9):1898-906.

4. Nash P, McInnes IB, Mease PJ, et al. Secukinumab versus adalimumab for psoriatic arthritis: comparative effectiveness up to 48 weeks using a matchingadjusted indirect comparison. Rheumatol Ther. 2018;5(1):99-122.

5. Ogdie A, Coates L. The changing face of clinical trials in psoriatic arthritis. Curr Rheumatol Rep. 2017;19(4):21.

6. Langley RG, Elewski BE, Lebwohl M, et al. Secukinumab in plaque psoriasis-results of two phase 3 trials. N Engl J Med. 2014;371(4):326-38.

7. Blauvelt A, Prinz JC, Gottlieb AB, et al. Secukinumab administration by pre-filled syringe: efficacy, safety and usability results from a randomized controlled trial in psoriasis (FEATURE). Br J Dermatol. 2015;172(2):484-93.

8. Paul C, Lacour JP, Tedremets L, et al. Efficacy, safety and usability of secukinumab administration by autoinjector/pen in psoriasis: a randomized, controlled trial (JUNCTURE). J Eur Acad Dermatol Venereol. 2015;29(6):1082-90.

9. Mease PJ, McInnes IB, Kirkham B, et al. Secukinumab inhibition of interleukin-17A in patients with psoriatic arthritis. N Engl J Med. 2015;373(14): 1329-39.

10. McInnes IB, Mease PJ, Kirkham B, et al. Secukinumab, a human anti-interleukin-17A monoclonal antibody, in patients with psoriatic arthritis (FUTURE 2): a randomised, double-blind, placebocontrolled, phase 3 trial. Lancet. 2015;386(9999): 1137-46.

11. Baeten D, Sieper J, Braun J, et al. Secukinumab, an interleukin-17A inhibitor, in ankylosing spondylitis. N Engl J Med. 2015;373(26):2534-48.

12. Deodhar A, Blanco R, Dokoupilová E, et al. Secukinumab improves signs and symptoms of non-radiographic axial spondyloarthritis: primary results of a randomized controlled phase III study. Arthritis Rheumatol. 2020;73(1):110-20.

13. Orbai AM, McInnes IB, Coates LC, et al. Effect of secukinumab on the different GRAPPA-OMERACT core domains in psoriatic arthritis: a pooled analysis of 2049 patients. J Rheumatol. 2020;47(6):854-64.

14. Orbai AM, de Wit M, Mease P, et al. International patient and physician consensus on a psoriatic arthritis core outcome set for clinical trials. Ann Rheum Dis. 2017;76(4):673-80.

15. Orbai AM, de Wit M, Mease PJ, et al. Updating the psoriatic arthritis (PsA) core domain set: a report from the PsA workshop at OMERACT 2016. J Rheumatol. 2017;44(10):1522-8.

16. McInnes IB, Mease PJ, Schett G, et al. Secukinumab provides rapid and sustained pain relief in psoriatic arthritis over 2 years: results from the FUTURE 2 study. Arthritis Res Ther. 2018;20(1):113.

17. Nash P, Mease PJ, McInnes IB, et al. Efficacy and safety of secukinumab administration by autoinjector in patients with psoriatic arthritis: results from a randomized, placebo-controlled trial (FUTURE 3). Arthritis Res Ther. 2018;20(1):47.

18. Kivitz A, Nash P, Tahir H, et al. Arthritis: primary results through 52 weeks from a phase- 3 randomized placebo-controlled study (FUTURE 4). J Clin Rheumatol. 2018;24(S3):364. 
19. Mease $P$, van der Heijde $D$, Landewé $R$, et al. Secukinumab improves active psoriatic arthritis symptoms and inhibits radiographic progression: primary results from the randomised, double-blind, phase III FUTURE 5 study. Ann Rheum Dis. 2018;77(6):890-7.

20. Baraliakos X, Gossec L, Pournara E, et al. Secukinumab in patients with psoriatic arthritis and axial manifestations: results from the double-blind, randomised, phase 3 MAXIMISE trial. Ann Rheum Dis. 2020. https://doi.org/10.1136/annrheumdis-2020218808.

21. Healy PJ, Helliwell PS. Measuring clinical enthesitis in psoriatic arthritis: assessment of existing measures and development of an instrument specific to psoriatic arthritis. Arthritis Rheum. 2008;59(5): 686-91.

22. Helliwell PS, Firth J, Ibrahim GH, Melsom RD, Shah I, Turner DE. Development of an assessment tool for dactylitis in patients with psoriatic arthritis. J Rheumatol. 2005;32(9):1745-50.

23. Langley RG, Feldman SR, Nyirady J, van de Kerkhof $\mathrm{P}$, Papavassilis C. The 5-point Investigator's Global Assessment (IGA) scale: a modified tool for evaluating plaque psoriasis severity in clinical trials. J Dermatolog Treat. 2015;26(1):23-31.

24. Fries JF, Spitz P, Kraines RG, Holman HR. Measurement of patient outcome in arthritis. Arthritis Rheum. 1980;23(2):137-45.

25. Mease PJ, Woolley JM, Bitman B, Wang BC, Globe DR, Singh A. Minimally important difference of Health Assessment Questionnaire in psoriatic arthritis: relating thresholds of improvement in functional ability to patient-rated importance and satisfaction. J Rheumatol. 2011;38(11):2461-5.

26. Husted JA, Gladman DD, Farewell VT, Long JA, Cook RJ. Validating the SF-36 health survey questionnaire in patients with psoriatic arthritis. J Rheumatol. 1997;24(3):511-7.

27. Kosinski M, Zhao SZ, Dedhiya S, Osterhaus JT, Ware JE Jr. Determining minimally important changes in generic and disease-specific health-related quality of life questionnaires in clinical trials of rheumatoid arthritis. Arthritis Rheum. 2000;43(7):1478-87.

28. Strand V, Burmester GR, Ogale S, Devenport J, John A, Emery P. Improvements in health-related quality of life after treatment with tocilizumab in patients with rheumatoid arthritis refractory to tumour necrosis factor inhibitors: results from the 24-week randomized controlled RADIATE study. Rheumatology (Oxford). 2012;51(10):1860-9.
29. Strand V, Kremer J, Wallenstein G, et al. Effects of tofacitinib monotherapy on patient-reported outcomes in a randomized phase 3 study of patients with active rheumatoid arthritis and inadequate responses to DMARDs. Arthritis Res Ther. 2015;17: 307.

30. Cella D, Wilson H, Shalhoub $\mathrm{H}$, et al. Content validity and psychometric evaluation of Functional Assessment of Chronic Illness Therapy-Fatigue in patients with psoriatic arthritis. J Patient Rep Outcomes. 2019;3(1):30.

31. Chandran V, Bhella S, Schentag C, Gladman DD. Functional assessment of chronic illness therapyfatigue scale is valid in patients with psoriatic arthritis. Ann Rheum Dis. 2007;66(7):936-9.

32. Tillett W, Lin CY, Zbrozek A, Sprabery AT, Birt J. A threshold of meaning for work disability improvement in psoriatic arthritis measured by the Work Productivity and Activity Impairment Questionnaire. Rheumatol Ther. 2019;6(3):379-91.

33. Cohen J. Statistical power analysis for the behavioral sciences. 2nd edn. New York: Routledge; 1988.

34. Costa L, Perricone C, Chimenti MS, et al. Switching between biological treatments in psoriatic arthritis: a review of the evidence. Drugs R\&D. 2017;17(4): 509-22.

35. Merola JF, Lockshin B, Mody EA. Switching biologics in the treatment of psoriatic arthritis. Semin Arthritis Rheum. 2017;47(1):29-37.

36. Mease PJ, Karki C, Liu M, et al. Discontinuation and switching patterns of tumour necrosis factor inhibitors (TNFis) in TNFi-naive and TNFi-experienced patients with psoriatic arthritis: an observational study from the US-based Corrona registry. RMD Open. 2019;5(1):e000880.

37. Moeller B, Papagiannoulis E, Christ L, et al. Drug retention of biological DMARDs targeting IL-12/23 or IL-17 versus TNF inhibitors, after a first line TNF inhibitor, in patients with psoriatic arthritis-an analysis in the Swiss SCQM Register [abstract]. Arthritis Rheumatol. 2019;71.

38. McInnes IB, Behrens F, Mease PJ, et al. Secukinumab versus adalimumab for treatment of active psoriatic arthritis (EXCEED): a double-blind, parallel-group, randomised, active-controlled, phase $3 \mathrm{~b}$ trial. Lancet. 2020;395(10235):1496-505.

39. Mease PJ, Smolen JS, Behrens F, et al. A head-tohead comparison of the efficacy and safety of ixekizumab and adalimumab in biological-naïve patients with active psoriatic arthritis: 24-week results of a randomised, open-label, blinded-assessor trial. Ann Rheum Dis. 2020;79(1):123-31. 
40. Sunkureddi P, Latremouille-Viau D, Meiselbach MK, Xie J, Hur P, Joshi R. Characteristics of patients with psoriatic arthritis receiving secukinumab and reasons for initiation: a US retrospective medical chart review. Rheumatol Ther. 2019;6(1):89-100.
41. Oelke KR, Chambenoit O, Majjhoo AQ, Gray S, Higgins K, Hur P. Persistence and adherence of biologics in US patients with psoriatic arthritis: analyses from a claims database. J Comp Eff Res. 2019;8(8):607-21. 